\title{
Fuzzy based subcutaneous fat assessment in real time ultrasound images
}

\author{
Pedro Couto ${ }^{1}$ Severiano Silva $^{2}$ Edurne Barrenechea $^{3}$ Ana Santos $^{2}$ Pedro Melo-Pinto $^{1}$ \\ ${ }^{1}$ CITAB Research Centre - UTAD University of Trás-os-Montes e Alto Douro, Portugal \\ ${ }^{2}$ CECAV Research Centre - UTAD University of Trás-os-Montes e Alto Douro, Portugal \\ ${ }^{3}$ UPNA Public University of Navarra, Spain
}

\begin{abstract}
The present work addresses the study of techniques of digital image processing based on fuzzy logic for subcutaneous fat (SF) estimation in real time ultrasound (RTU) images. The process for SF automatic estimation in RTU images is divided into two steps: the identification of the image area corresponding to the SF regions and, from these regions, the SF estimation. In order to deal with the ambiguity present in the gray levels of RTU images, fuzzy logic based methods are used. Experimental results are also presented.
\end{abstract}

Keywords: Atanassov's Intuitionistic Fuzzy Sets, Entropy, Multi-thresholding

\section{Introduction}

For animal science, reliable information and accurate subcutaneous fat (SF) measurements are required to evaluate body reserves of live animals [1]. Body reserves are decisive for nutritional, reproductive and health management [2]. Classically, measurements of body reserves have been done by direct or indirect calorimetry, comparative slaughter or dilution techniques [3]. This is a slow, tedious, and expensive process and, as direct consequence, tools for rapid, non-invasive and inexpensive estimation of body composition have been continuously pursued. Ultrasound provides a noninvasive method for estimating fat accretion and body composition on live animals $[2,4]$. Ultrasound imagery is a fast, reliable technology and provides a nondestructive way to predict body reserves by measuring subcutaneous fat under the skin $[2,5]$. Nevertheless, manually processing real time ultrasound (RTU) images is a non accurate, slow and tedious process. Despite the existence of a broad range of software for image analysis and visual inspection, the supply of these systems for the analysis of this kind of RTU images is still under provided. In this work, a digital image processing methodology for in vivo SF estimation in RTU images is presented.

In the image analysis methodologies issued in this work, the segmentation of the image plays a decisive role towards a good processed image data since, being the starting point of the process it can critically affect its performance. In this sense, the image is decomposed into meaningful parts for further analysis, resulting in the partition of the initial set of pixels in the image into a finite set of regions (subsets) according to a certain criterion. In the present case the purpose of the segmentation is to decompose the image into two separate regions: the region with pixels belonging to the $\mathrm{SF}$ and a second region corresponding to all the non SF regions. In order to achieve such result, due to the difficulties imposed by the intrinsic characteristics of the issued RTU images, rather than a bi-level thresholding approach, a tri-level thresholding approach was implemented. This tri-level thresholding approach results in only two (instead of three) image regions since the image binarization is made using only the higher threshold value of the two computed thresholds.

Extensive research has been conducted in the image segmentation research field, and many types of image segmentation approaches have been proposed in the literature, each one of them based on a certain methodology to classify the regions $[6,7,8,9,10,11,12]$.

Considering fuzzy set theory as an efficient tool for image segmentation, within this theory, the most popular algorithms are those that use the concept of fuzzy entropy $[13,14,15,16,17,18]$. In this work, Atanassov's intuitionistic fuzzy sets $[19,20]$ are employed in such way that Atanassov's intuitionistic index $(\pi)$ is used to quantify the ambiguity present in the image pixels and, intuitionistic entropy is used in the same sense as fuzzy entropy is used in fuzzy logic based segmentation algorithms.

The presented segmentation approach was developed within the work presented in [21] where the main goal is to perform fuzzy logic based analysis in ambiguous data images. The proposed approach is an evolution/extension of the methodology, based on Atanassov's intuitionistic fuzzy sets (A-IFSs) presented in [21], that uses a tri-level thresholding approach. This tri-level approach was experimentally selected since this approach was the one that accurately segmented the SF region. In this work, besides the segmentation methodology, a method for quantification of the SF is also presented.

\section{Proposed Methodology}

Following the work in progress in animal science, in this work we used an experimental group consisting of 10 garrano horses (290 $\pm 10 \mathrm{~kg}$ body weight). Their body condition was evaluated using RTU images [22] obtained at the lumbar site using Aloka SSD 500V equip- 
ment (Aloka, Tokyo, Japan) equiped with a $5.0 \mathrm{MHz}$ probe. In Fig. 1 an example of an RTU image and its corresponding $\mathrm{SF}$ region is presented.

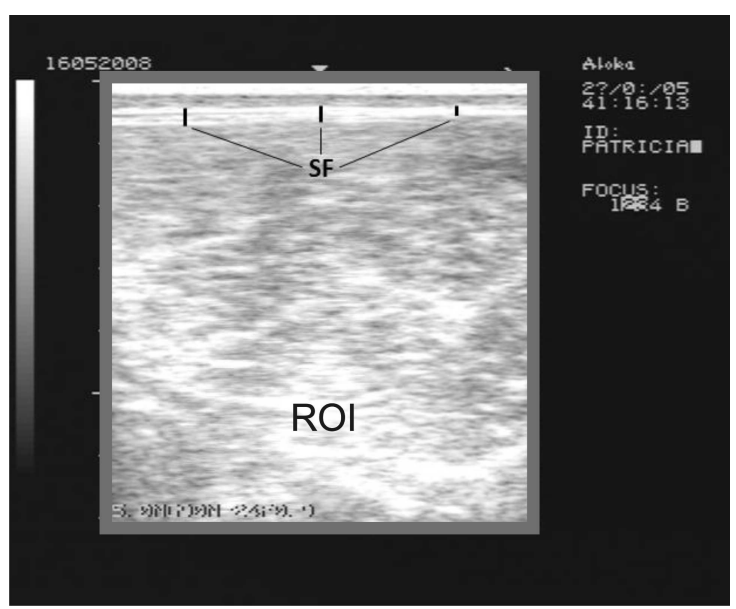

Figure 1: RTU image with corresponding ROI and SF region.

In Fig. 1 the region of interest (ROI) of the RTU image is also pointed out since the used thresholding approach only takes into account pixels belonging to the ROI.

Up until now, in animal science, the experts needed to process the RTU images manually. This manual approach (MI) is illustrated in Fig. 1 where one can see the three arbitrarily chosen measure points from which average the expert calculates the estimated SF thickness.

This processing of the RTU images besides being slow and tedious is, most of all, neither accurate nor reproducible. We think there are two main reasons for this lack of accuracy: (i) the inherent ambiguity of the RTU images that makes it very difficult to determine the $\mathrm{SF}$ region boundaries and (ii) the nature of the measurement procedure that involves choosing three arbitrary measurement points. Our main goal with this work is to overcome both of these difficulties.

After experimental tests made with the aid of an animal science expert, a tri-level approach of thresholding ( 2 threshold values, $t 1$ and $t 2$ such that $t 1<t 2$ ) was used in this work. This tri-level thresholding approach that would normally give raise to 3 image regions results only into two image regions since the image binarization is made using only the threshold $t 2$ in the following way:

$$
q(x, y)=\left\{\begin{array}{lll}
0 & \text { if } & q(x, y) \leq t 2 \\
L-1 & \text { if } & q(x, y)>t 2
\end{array}\right.
$$

being $L$ the gray levels of an image $Q$.

Finally, after the image binarization, a method for the $\mathrm{SF}$ region quantification was also developed.

\subsection{Atanassov's intuitionistic fuzzy sets based image tri-level thresholding}

In the present approach, being a tri-level thresholding approach, the membership of the pixel to each one of the three image objects starts being represented by three fuzzy sets, each one associated with one of the three image regions $(R 1, R 2$ or $R 3)$. In order to choose/construct the membership function of each pixel of the image to the associated fuzzy set, four numerical values are assigned to each one of them:

- Three values for representing the expert knowledge of the membership of the pixel to each one of the three image objects. Three membership functions, constructed by the expert using dissimilarity functions(see [23]), are used to obtain each one of these values.

- The expert knowledge/ignorance, in determining the above mentioned membership functions, is represented by a third value obtained through Atanassov's intuitionistic index $(\pi)$.

This fourth value $(\pi)$ indicates how sure the expert is in assigning each pixel to the correspondent region, being that if $\pi=0$ then the expert is positively sure of the pixel's belonging. This value increases with respect to the lack of knowledge/ignorance of the expert as to whether the pixel belongs to $R 1, R 2$ or $R 3$ reaching to the value of 1 when the expert used the greatest lack of knowledge/ignorance/intuition allowed in the assigning of the pixel to the regions (i.e., the construction of the membership functions of the set associated with that pixel), resulting in a Atanassov's intuitionistic fuzzy index maximum value. For this reason, A-IFSs $[19,20]$ are used.

Finally, after calculating the entropy values of each one of the L A-IFSs associated with the image, the combination of $t 1, t 2 \in\{0,1, \cdots, L-1\}$ such that $t 1<t 2$, associated with the A-IFS of lowest entropy are chosen as the best thresholds. The justification for this choice is given in [21] where, since we've used the entropy proposed by Burillo and Bustince (see [24]), it is proven that this entropy will be null when the set is a FSs and will be maximum when the set is totally intuitionistic.

As it has been said before, the final outcome of this tri-level thresholding methodology is a binary image obtained by setting all pixels $q(x, y) \leq t 2$ to 0 and all the other to $L-1$.

Being $(x, y)$ the coordinates of each pixel on the image $Q$, and being $q(x, y)$ the gray level of the pixel $(x, y)$ so that $0 \leq q(x, y) \leq L-1$ for each $(x, y) \in Q$ where $L$ is the image grayscale, the implementation of this methodology can be described as follows:

\subsubsection{Fuzzy sets construction}

Considering an image $Q$ and two intensity thresholds $t 1$ and $t 2$, we will construct $L$ fuzzy sets $\tilde{Q}_{O 1 t}$ associated with region $R 1, L$ fuzzy sets $\tilde{Q}_{O 2 t}$ associated with region $R 2$, and another $L$ fuzzy sets $\tilde{Q}_{O 3 t}$ associated with region $R 3$, in such way that the membership function of each element to the sets $\tilde{Q}_{O 1 t}, \tilde{Q}_{O 2 t}$ and $\tilde{Q}_{O 3 t}$ must express the relationship between the intensity $q$ of the pixel and its membership to region $R 1, R 2$ or $R 3$ respectively.

For each possible combinations of $t 1, t 2 \in$ $\{0,1, \cdots, L-1\}$, such that $t 1<t 2$, the mean of the 
intensities of gray of the pixels that belong to the region $R 1\left(m_{O 1 t}\right)$, the mean of the intensities of gray of the pixels that belong to the region $R 2\left(m_{O 2 t}\right)$, and the mean of the intensities of gray of the pixels that belong to the region $R 3\left(m_{O 3 t}\right)$ are given by the following expressions:

$$
\begin{gathered}
m_{O 1 t}=\frac{\sum_{q=0}^{t 1} q h(q)}{\sum_{q=0}^{t 1} h(q)}, \\
m_{O 2 t}=\frac{\sum_{q=t 1+1}^{t 2} q h(q)}{\sum_{q=t 1+1}^{t 2} h(q)}, \\
m_{O 3 t}=\frac{\sum_{q=t 2+1}^{L-1} q h(q)}{\sum_{q=t 2+1}^{L-1} h(q)} .
\end{gathered}
$$

With $h(q)$ being the number of pixels of the image with intensity $q$.

We construct the membership functions of each possible combinations of intensities $t 1, t 2$ in the above mentioned conditions, to the sets $\tilde{Q}_{O 1 t}, \tilde{Q}_{O 2 t}$ and $\tilde{Q}_{O 3 t}$ in the following way [25]:

$$
\begin{aligned}
& \mu_{\tilde{Q}_{O 1 t}}(q)=F\left(d\left(\frac{q}{L-1}, \frac{m_{O 1 t}}{L-1}\right)\right), \\
& \mu_{\tilde{Q}_{O 2 t}}(q)=F\left(d\left(\frac{q}{L-1}, \frac{m_{O 2 t}}{L-1}\right)\right), \\
& \mu_{\tilde{Q}_{O 3 t}}(q)=F\left(d\left(\frac{q}{L-1}, \frac{m_{O 3 t}}{L-1}\right)\right) .
\end{aligned}
$$

In this approach we use the function $F(x)=1-0.5 x$ along with the restricted dissimilarity function $d(x, y)=$ $|x-y|$ which conduct us to the fuzzy sets $\tilde{Q}_{O 1 t}, \tilde{Q}_{O 2 t}$ and $\tilde{Q}_{O 3 t}$ represented by the following membership functions:

$$
\begin{aligned}
& \mu_{\tilde{Q}_{O 1 t}}(q)=1-0.5\left|\frac{q}{L-1}-\frac{m_{O 1 t}}{L-1}\right|, \\
& \mu_{\tilde{Q}_{O 2 t}}(q)=1-0.5\left|\frac{q}{L-1}-\frac{m_{O 2 t}}{L-1}\right|, \\
& \mu_{\tilde{Q}_{O 3 t}}(q)=1-0.5\left|\frac{q}{L-1}-\frac{m_{O 3 t}}{L-1}\right| .
\end{aligned}
$$

Note that $F(x)$ and $d(x, y)$ are only ones of the set of possibilities of functions that could have been used (see [21]). The constructed membership functions are always greater than or equal to 0.5 and, the smaller the distance between a pixel's intensity $q$ and the mean of intensities of the considered region (region $R 1, R 2$ or $R 3)$, the greater the value of its membership to that region.

\subsubsection{Atanassov's intuitionistic index $\pi$ quantification}

In this approach we interpret Atanassov's intuitionistic index $\pi$ as the lack of knowledge/ignorance of the expert in assigning the membership value of a certain pixel to the regions $R 1, R 2$ or $R 3$ of the image. Under this interpretation of $\pi$, we will consider that $\mu_{\tilde{Q}_{O 1 t}}, \mu_{\tilde{Q}_{O 2 t}}$ and
$\mu_{\tilde{Q}_{O 3 t}}$ indicates the expert's degree of knowledge of the pixel belonging to region $R 1, R 2$ or $R 3$ respectively.

In any case the following conditions must be fulfilled:

1. The lack of knowledge that the expert uses in the choice of the membership of a pixel must be zero if he is certain that the pixel belongs to one of the considered regions.

2. The lack of knowledge/ignorance must decrease with respect to the certainty of the expert as to the pixel belonging to one of the regions.

3. The lack of knowledge/ignorance must have the lower possible influence on the choice of the membership degree. In the worst of cases, the lack of knowledge will have a maximum influence of 50 percent.

In this context, $\pi(q)$ is the quantification of the lack of knowledge/ignorance of the expert in the selection of the membership functions $\mu_{\tilde{Q}_{O 1 t}}(q), \mu_{\tilde{Q}_{O 2 t}}(q)$ and $\mu_{\tilde{Q}_{O 3 t}}(q)$.

We used the following expression for $\pi(q)$ :

$\pi(q)=\wedge\left(1-\mu_{\tilde{Q}_{O 1 t}}(q), 1-\mu_{\tilde{Q}_{O 2 t}}(q), 1-\mu_{\tilde{Q}_{O 3 t}}(q)\right)$

The expression 1 fulfils the above mentioned conditions since, $0.5 \leqslant \mu_{\tilde{Q}_{O 1 t}}(q) \leqslant 1,0.5 \leqslant \mu_{\tilde{Q}_{O 2 t}}(q) \leqslant 1$ and $0.5 \leqslant \mu_{\tilde{Q}_{O 3 t}}(q) \leqslant 1$ then:

$$
\begin{gathered}
\pi(q)=0 \quad \text { if and only if } \\
\mu_{\tilde{Q}_{O 1 t}}(q)=1 \text { or } \mu_{\tilde{Q}_{O 2 t}}(q)=1 \text { or } \mu_{\tilde{Q}_{O 3 t}}(q)=1
\end{gathered}
$$

meaning that the expert is positively sure that the pixel belongs to one of the objects.

$$
\pi(q)=0.5 \quad \text { if and only if }
$$

$\mu_{\tilde{Q}_{O 1 t}}(q)=0.5$ and $\mu_{\tilde{Q}_{O 2 t}}(q)=0.5$ and $\mu_{\tilde{Q}_{O 3 t}}(q)=0.5$ meaning that the expert has the greatest lack of knowledge/ignorance in determining to which region the pixel belongs to.

Hence,

$$
0 \leqslant \pi(q) \leqslant 0.5
$$

\subsubsection{Atanassov's intuitionistic fuzzy sets construction}

In this section, for all possible combinations of $t 1, t 2 \in$ $\{0,1, \cdots, L-1\}$, such that $t 1<t 2$, we will associate an A-IFS (using the index $\pi$ described in the subsection above) with each one of the fuzzy sets $\tilde{Q}_{O 1 t}, \tilde{Q}_{O 2 t}$ and $\tilde{Q}_{O 3 t}$, in the following way:

$Q_{O 1 t}=\left\{\left(q, \mu_{Q_{O 1 t}}(q), \nu_{Q_{O 1 t}}(q)\right) \mid q=0,1, \cdots, L-1\right\}$,

given by

$$
\begin{aligned}
& \mu_{Q_{O 1 t}}(q)=\mu_{\tilde{Q}_{O 1 t}}(q) \\
& \nu_{Q_{O 1 t}}(q)=1-\mu_{Q_{O 1 t}}(q)-\pi(q)
\end{aligned}
$$

and

$Q_{O 2 t}=\left\{\left(q, \mu_{Q_{O 2 t}}(q), \nu_{Q_{O 2 t}}(q)\right) \mid q=0,1, \cdots, L-1\right\}$, 
given by

$$
\begin{aligned}
& \mu_{Q_{O 2 t}}(q)=\mu_{\tilde{Q}_{O 2 t}}(q) \\
& \nu_{Q_{O 2 t}}(q)=1-\mu_{Q_{O 2 t}}(q)-\pi(q)
\end{aligned}
$$

and

$Q_{O 3 t}=\left\{\left(q, \mu_{Q_{O 3 t}}(q), \nu_{Q_{O 3 t}}(q)\right) \mid q=0,1, \cdots, L-1\right\}$,

given by

$$
\begin{aligned}
& \mu_{Q_{O 3 t}}(q)=\mu_{\tilde{Q}_{O 3 t}}(q) \\
& \nu_{Q_{O 3 t}}(q)=1-\mu_{Q_{O 3 t}}(q)-\pi(q)
\end{aligned}
$$

\subsubsection{Entropy calculation}

At this step we are going to calculate, for each possible combinations of $t 1, t 2 \in\{0,1, \cdots, L-1\}$, such that $t 1<t 2$, the entropy $\varepsilon_{T}$ of each one of the $L$ intuitionistic fuzzy sets of Atanassov $Q_{O 1 t}, Q_{O 2 t}$ and $Q_{O 3 t}$. In this approach we use the Type 2 entropy defined by Burillo and Bustince by means of the following expression [24]:

$$
\varepsilon_{T_{2}}\left(Q_{O 1 t}\right)=\frac{1}{N \times M} \sum_{q=0}^{L-1} h(q) \cdot \pi(q)
$$

where $N \times M$ are the image dimensions in pixels, $h$ is the image histogram and $\pi$ is obtained with equation( 1 ).

\subsubsection{Threshold values selection}

The combination of the gray levels $t 1, t 2 \in$ $\{0,1, \cdots, L-1\}$ such that $t 1<t 2$, associated with the A-IFS of lowest entropy are chosen as the best possible thresholds.

\subsection{Segmentation results}

In Fig. 2, 10 RTU images corresponding to each one of the 10 garrano horses of the experimental group are shown.

Looking at Fig. 2 one can see that some images exhibit different characteristics, regarding their gray levels (some are lighter than others) when compared with each other. As a result, the SF region pixels gray levels are not constant within the set of images making the segmentation task more difficult.

In Fig. 3 the segmented images corresponding to the 10 images of Fig. 2 are shown.

The results presented in Fig. 3 show that the SF regions of all the images were properly segmented.

We've asked the animal science expert to process the segmented images, assisted approach (AI), in same way he did with the original ones using the MI approach. As expected, due to the ambiguous nature of the original RTU images, the results obtained by the animal science expert when processing the segmented images were more consistent and with much higher repeatability when compared with the ones obtained by the same expert with the classical MI approach. Moreover, the animal science expert was much more confident with the
A

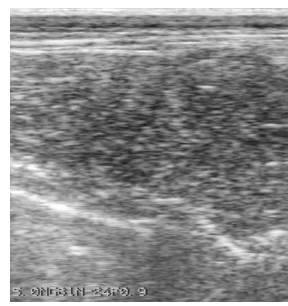

B

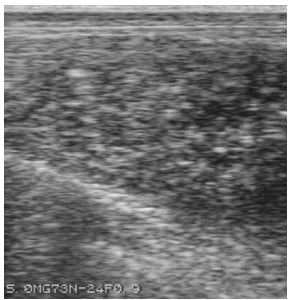

C

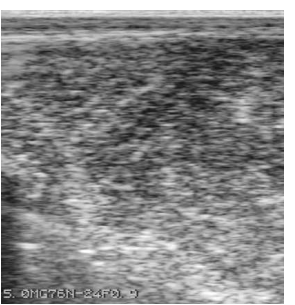

D

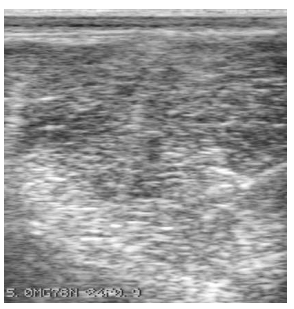

E

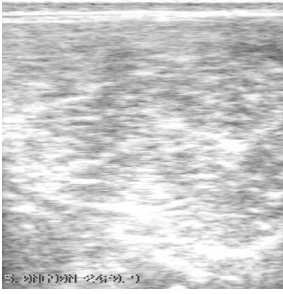

F
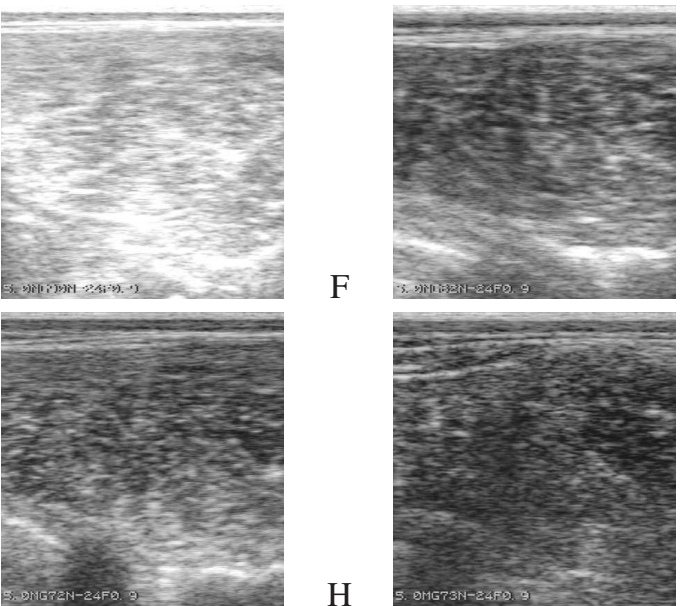

$\mathrm{H}$

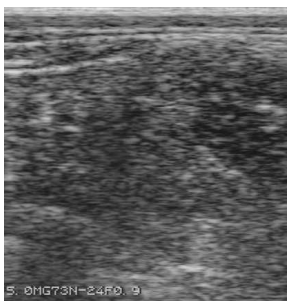

I
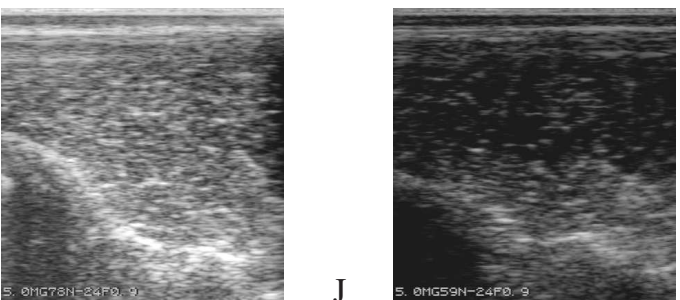

Figure 2: Original RTU images.

measurements obtained with the AI approach with relation to the ones he obtained with the manual approach. We believe that the main reason for this outcome is that using AI approach the ambiguity problem was overcome by the image segmentation.

\subsection{SF region quantification methodology}

Although the results for SF estimation obtained with the AI approach were more consistent and with high repeatability, due to the nature of the measurement process (manually select three points of the SF zone from which average the SF is assessed), if we change the animal science expert one could end up with less consistent results with much lower repeatability. For that reason, a fully automatic approach for SF estimation, based on the segmented images obtained was developed in order to establish a consistent SF estimation methodology where the accuracy of the results and their repeatability is assured making them reproducible by any animal science 
A

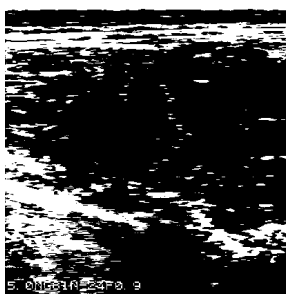

$\mathrm{C}$

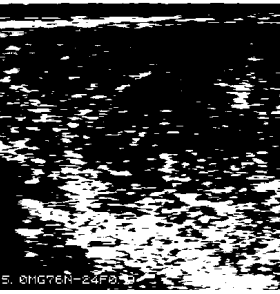

$\mathrm{E}$

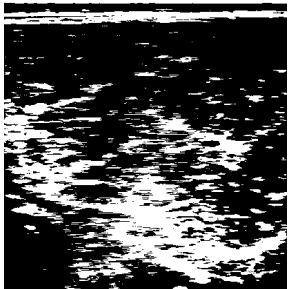

$\mathrm{G}$
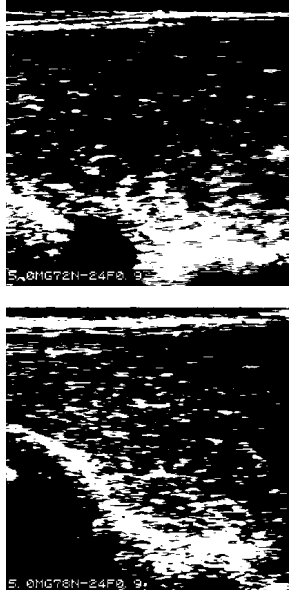

Figure 3: Segmented images using the proposed approach.

expert.

This automatic inspection use the line histograms $(H)$ of the segmented images. These line histograms are similar to classic luminance histogram in the way that they provide a statistical view on the distribution of pixels on the image and can be defined by the following expression:

$$
H(x)=\frac{\sum_{y=0}^{M} q(x, y)}{L-1}, \forall x \in[0, N]
$$

In Fig. 4 we present the line histograms of RTU images $\mathrm{A}, \mathrm{C}$ and $\mathrm{I}$ where the $\mathrm{SF}$ region correspond to the black region of the line histogram. In this sense to estimate the SF we proceeded to the measurement of the thickness, in pixels and later converted to $\mathrm{mm}$ $(10 \mathrm{~mm}: 60$ pixels according to the equipment calibration), of this region.

In this automatic approach (AuI) the region area was used to determine the SF thickness in the following way: first we experimentally established $n \alpha$ cuts $\left(\alpha_{1}, \alpha_{2}, \cdots\right.$
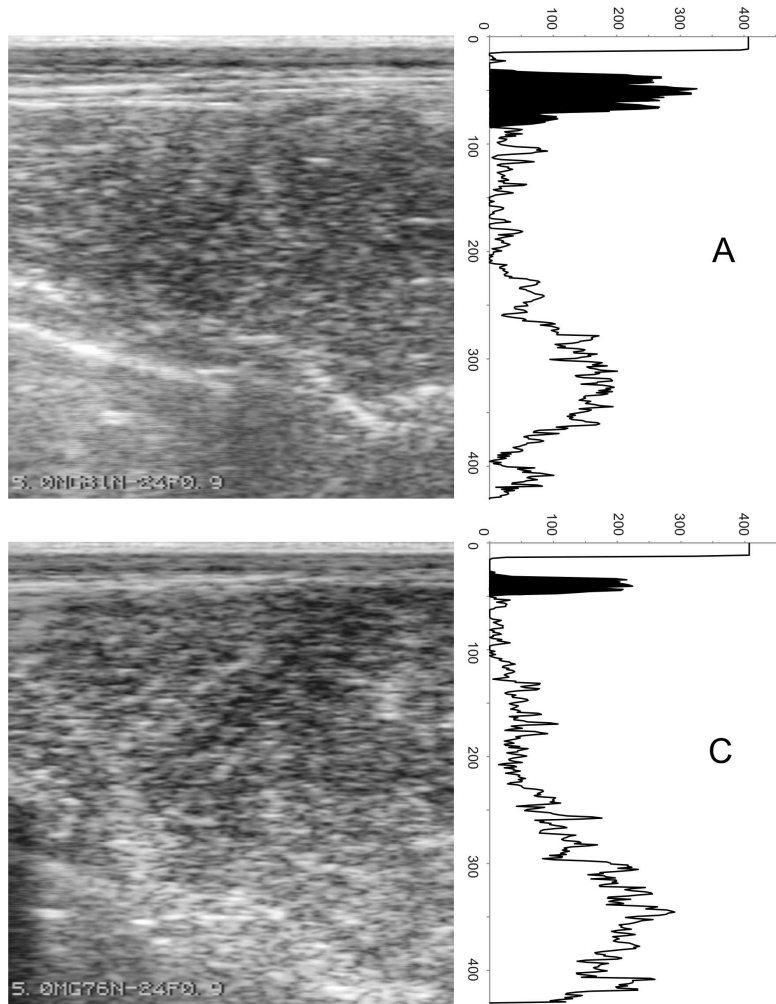

A
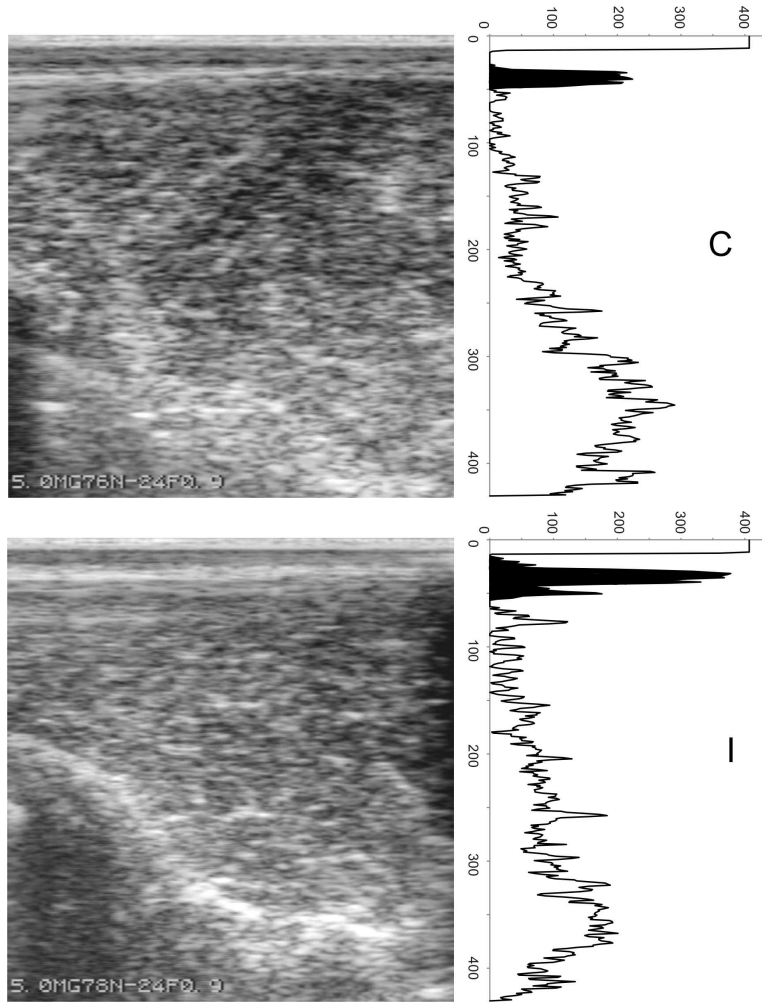

Figure 4: Original images and corresponding line histograms.

, $\alpha_{n}$ ) with $\alpha_{i}=i * 50$ pixels. The number of $\alpha$ cuts depends on the shape of the SF region on histogram. To provide a better understanding of this relation, in Fig. 5 we present an example of an SF region with four $\alpha$ cuts, i.e., $n=4$. Using the above mentioned $\alpha$ cuts, a collection of ordered lengths $L=\left\{l_{1}, l_{2}, \cdots, l_{n}\right\}$ with $l_{i} \in[0 ; 1]$, is obtained through the histogram in the following way:

$$
l_{i}=\frac{\text { last }_{i}-\text { first }_{i}}{N}
$$

being first $_{i}$ and last $t_{i}$ the intersections of the $\alpha$ cut $i$ with the line histogram and $N$ the same as in Eq. 2.

These lengths, due to the cumulative nature of the line histogram, are ordered by the amount and the feasibility of the information they provide. This way, the information provided by $l_{i-1}$ is more feasible than the one provided by $l_{i}$.

Then the optimal area (Aopt) of each one of the $n$ SF regions in the line histogram is calculated by means of the following expression: 


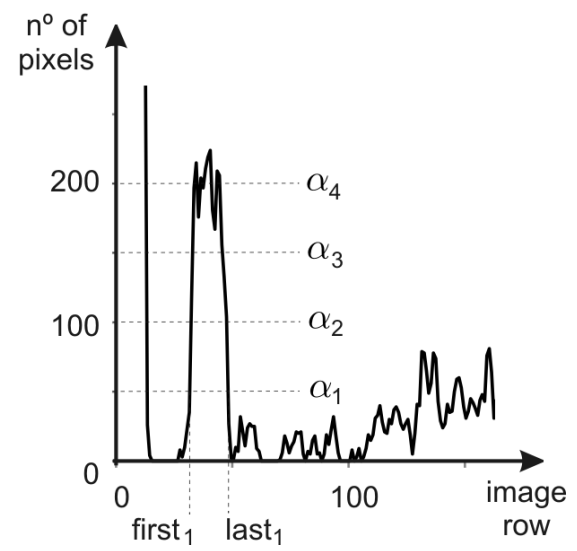

Figure 5: SF line histogram zone $\alpha$ cuts.

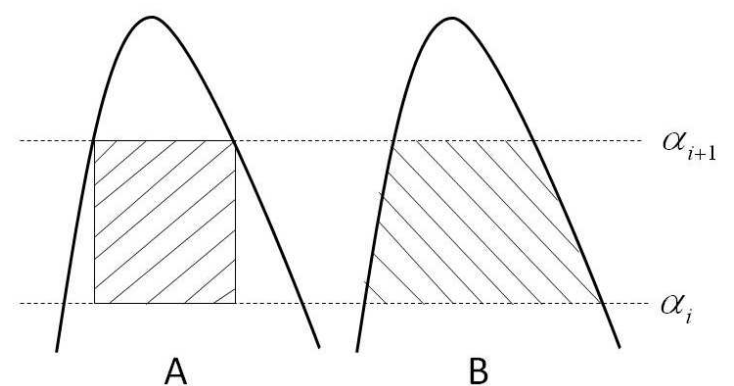

Figure 6: Representation of the optimal and real areas. A - Aopt and B - Areal

$$
\text { Aopt }_{i}=l_{i} * \alpha_{i} \quad \text { for all } \quad i \in\{1, \cdots, n\}
$$

The real area (Areal) of each one of the four SF regions in the line histogram is also calculated:

$$
\text { Areal }_{i}=\sum_{x=\text { first }_{i}}^{\text {last }_{i}} A(x)
$$

where

$$
\begin{gathered}
A(x)= \begin{cases}H(x)-\alpha_{i-1} & \text { if } \quad H(x)<\alpha_{i} \\
\alpha_{i}-\alpha_{i-1} & \text { otherwise }\end{cases} \\
\text { for all } \quad i \in\{1, \cdots, n\}
\end{gathered}
$$

For better understanding, in Fig. 6 a representation of the areas Aopt and Areal is shown.

Before calculating the thickness of the SF zone, each one of the $n$ zones is weighted by means of a set of weights $W=\left\{w_{1}, w_{2}, \cdots, w_{n}\right\}$ with $w_{i} \in[0,1]$, in the following way:

$$
\begin{aligned}
& w_{i}=\frac{\text { Areal }_{i}}{n * \text { Aopt }_{i} * \sum_{i=1}^{n} \frac{\text { Areal }_{i}}{\text { Aopt }_{i}}} \\
& \text { for all } \quad i \in\{1, \cdots, n\}
\end{aligned}
$$

This way, all weights lie in the unit interval and sum up to 1 and the thickness of the SF area is calculated using an Ordered Weighted Averaging (OWA) operator $[26,27,28,29]$ according to the following expression:

$$
S F=\sum_{i=1}^{n} l_{i} * w_{i}
$$

Finally, the SF thickness is converted to $m m$ using the following expression:

$$
S F_{m m}=S F * \frac{N}{6}
$$

In Table 1 the numerical values of the SF estimated thickness obtained with the three approaches issued in this work (MI, AI and AuI) are presented.

Table 1: Numerical values of SF thickness measures

\begin{tabular}{cccc}
\hline & MI & AI & AuI \\
\hline A & 4.08 & 5.56 & 6.44 \\
B & 3.11 & 4.00 & 6.29 \\
C & 2.28 & 2.09 & 2.13 \\
D & 2.73 & 2.37 & 1.93 \\
E & 2.00 & 2.37 & 2.66 \\
F & 3.83 & 2.89 & 3.55 \\
G & 3.50 & 3.06 & 2.90 \\
H & 3.50 & 4.48 & 4.58 \\
I & 2.22 & 2.61 & 2.53 \\
J & 2.50 & 1.93 & 1.23 \\
\hline
\end{tabular}

In Table 2 we statistically evaluate the performance of the proposed methodology with relation to the other two methodologies for SF estimation issued in this work: the classical manual methodology (Manual Inspection - MI), the semi-automatic method (Assisted Inspection - AI) and the automatic method (Automatic Inspection - AuI). Keep in mind that: the MI approach uses the original RTU images of Fig. 1 and both AI and AuI approaches use the segmented images Fig. 3 and, the MI and AI approaches need the presence of the an animal science expert.

Table 2: Correlation matrix of SF thickness measures

\begin{tabular}{cccc} 
& MI & AI & AuI \\
\hline MI & 1 & $0.759^{* *}$ & $0.705^{*}$ \\
AI & & 1 & $0.919^{* *}$ \\
AuI & & & 1 \\
\hline$p<0.05^{*} ; p<0.01^{* *}$ & & &
\end{tabular}

The correlation analysis made in Table 2 shows a strong relation $(p<0.01)$ between the AI and AuI approaches. Nevertheless, we are aware that since this study was made in live animals, this is not a fully objective analysis because the real SF thickness measurement values are not available.

\section{Conclusions}

Aware of the characteristics of RTU images (high level of ambiguity in the levels of gray) a image segmentation methodology based on fuzzy logic was used. In 
the proposed automatic method, the human error factor is removed and, most of all, results repeatability is ensured. Both these problems were unsolved until now in the animal science field. The human error is removed primarily by means of the image segmentation procedure that accurately separates the SF region taking into account the pixels predicate (the predicate needed by a pixel to belong to the SF region) ambiguity by means of the Atanassov's intuitionistic fuzzy index $(\pi)$. Results repeatability is ensured by means of the proposed automatic SF region quantification procedure that, making use of the SF region line histogram and an ordered weighted average operator, able us to accurately quantify the SF region.

Moreover, the analysis of a large number of images is facilitated. This work provides a reliable methodology for analyzing RTU images, that can be used to monitor the body composition of horses.

It's our belief that the proposed application is a step forward for the animal science research field allowing a quick and accurate estimation of animal body composition namely, animal subcutaneous fat. Further work is intended, focusing on the adaptation of the proposed analysis methodology to other parameters of interest in this type of images.

\section{References}

[1] J. Bewley and M. Schutz. An interdisciplinary review of body condition scoring for dairy cattle. Prof. Animal Science, 24:507-529, 2008.

[2] U. Schröder and R. Staufenbiel. Methods to determine body fat reserves in the dairy cow with special regard to ultrasonographic measurement of backfat thickness. Journal of Dairy Science, 89:114, 2006.

[3] P. McDonald, R. Edwards, J. Greenhalgh, and C. Morgan. Animal Nutrition. Sixth Edition. Prentice-Hall, 2002.

[4] J. Stouffer. History of ultrasound in animal science. Journal of Ultrasound in Medicine, 23:577584, 2004.

[5] L. Gentry, D. Thompson, G. Gentry, R. Del Vecchio, K. Davis, and P. Del Vecchio. The relationship between body condition score and ultrasonic fat measurements in mares of high versus low body condition. Journal of Equine Veterinary Science, 24:198-203, 2004.

[6] V. Jawahar, K. Biswas, and K. Ray. Analysis of fuzzy thresholding schemes. Pattern Recognition, 33:1339-1349, 2000.

[7] M. Sezgin and B. Sankur. Survey over image thresholding techniques and quantitative performance evaluation. Journal of Electronic Imaging, 13(1):146-165, January 2004.

[8] K. Fu and J. Mui. A survey on image segmentation. Pattern Recognition, 13:3-16, 1981.

[9] R. Haralick and L. Shapiro. Image segmentation techniques. Computer Vision, Graphics and Image Processing, 29:100-132, 1985.
[10] N. Pal and S. Pal. A review on image segmentation techniques. Pattern Recognition, 26:1277-1294, 1993.

[11] D. Benboudjema and W. Pieczynski. Unsupervised image segmentation using triplet markov fields. Computer Vision and Image Understanding, 99:476-498, 2005.

[12] N. Lopes, P. Couto, H. Bustince, and P. MeloPinto. Automatic histogram threshold using fuzzy measures. IEEE Transactions on Image Processing, 19(1):199-204, 2010.

[13] L. Huang and M. Wang. Image thresholding by minimizing the measure of fuzziness. Pattern recognition, 28(1):41-51, 1995.

[14] J. Bezdek, J. Keller, R. Krisnapuram, and N. Pal. Fuzzy Models and algorithms for pattern recognition and image processing, The Handbooks of Fuzzy Sets Series. Kluwer Academic Publishers, Boston/London/Dordrecht, 1999.

[15] M. Forero. Fuzzy Filters for Image Processing, chapter Fuzzy thresholding and histogram analysis, pages 129-152. Springer, 2003.

[16] H. Bustince, M. Pagola, E. Barrenechea, J. Fernandez, P. Melo-Pinto, P. Couto, H. Tizhoosh, and Montero J. Ignorance functions. an application to the calculation of the threshold in prostate ultrasound images. Fuzzy Sets and Systems, 161(1):2036, 2010.

[17] P. Couto, H. Bustince, A. Jurio, G. Gluhchev, A. Varejao, and P. Melo-Pinto. A-ifss based iterative thresholding in gait analysis. IEEE IS 2010 IEEE International Conference on Intelligent Systems Proceedings, pages 270-275, 2010.

[18] P. Couto, H. Bustince, M. Pagola, A. Jurio, and P. Melo-Pinto. A-ifss entropy based image multi-thresholding. Trends in Applied Intelligent Systems: Lecture Notes in Computer Science, 6098:341-349, 2010.

[19] K. Atanassov. Intuitionistic fuzzy sets. Fuzzy Sets and Systems, 20:87-96, 1986.

[20] K. Atanassov. Intuitionistic fuzzy sets. Theory and applications. Springer-Verlag, Berlin Heidelberg New York, 1 edition, January 1999.

[21] H. Bustince, M. Pagola, P. Melo-Pinto, E. Barrenechea, and P. Couto. Fuzzy Sets and Their Extensions: Representation, Aggregation and Models, in Studies in Fuzziness and Soft Computing, chapter Image threshold computation by modelizing knowledge/unknowledge by means of A-IFSs, pages 627-644. Springer-Verlag, Berlin Heidelberg New York, 2007.

[22] A. Santos, E. Verhees, S. Silva, M. Quaresma, V. Sousa, and W. Pellikan. Morphological body measurements, body condition score and ultrasound measurements of portuguese garrano horse breed. Proceedings of the 60th Annual Meeting of the European Association for Animal Production, page 422, 2009.

[23] E. Barrenechea. Image Thresholding with Intervalvalued Fuzzy Sets. Edge Detection. Contrast. $\mathrm{PhD}$ 
thesis, Universidad Pública de Navarra, 2005.

[24] P. Burillo and H. Bustince. Entropy on intuitionistic fuzzy sets and on intervalvalued fuzzy sets. Fuzzy Sets and Systems, 78:81-103, 1996.

[25] H. Bustince, E. Barrenechea, and M. Pagola. Restricted equivalence functions. Fuzzy Sets and Systems, 157:2333-2346, 2006.

[26] R. Yager. On ordered weighted averaging aggregation operators in multicriteria decisionmaking. IEEE Transactions on Systems, Man and Cybernetics, 18(1):183-190, 1988.

[27] R. Yager. Applications and extensions of owa aggregations. International Journal Man-Machine Studies, 37:103-132, 1992.

[28] R. Yager and J. Kacprzyk. The Ordered Weighted Averaging Operators: Theory and Applications. Kluwer Academic Publishers, 1997.

[29] X. Liu. Some properties of the weighted owa operator. IEEE Transactions on Systems, Man and Cybernetics, Part B: Cybernetics, 36(1):118-127, 2006. 